\title{
AN INTEGRAL IN TOPOLOGICAL SPACES ${ }^{1}$
}

\author{
BY W. F. PFEFFER
}

\author{
Communicated by J. B. Diaz, October 2, 1968
}

A definition of an integral by majorants and minorants was first given by Perron in [8] for functions of one real variable. Perron's definition was generalized by Bauer in [1] and later on by Mařík in [7], for the case of functions of several real variables whose domains of definition are compact intervals. This generalization, however, was not sufficient, because no adequate substitution theorem holds: even a very simple transformation, e.g., a rotation, of a compact interval is not necessarily a compact interval again. A generalization which admits a relatively suitable substitution theorem was given by the author in [9] and [10]. Although the integral there is defined in a locally compact first countable Hausdorff space, the main emphases are on applications to Euclidean spaces; also only functions with compact domains of definition are integrated.

In this paper we shall define a Perron-like integral in an arbitrary topological space and without any restrictions on the domains of integrable functions. Such generality, of course, causes some changes in basic definitions. Because of omission of the first axiom of countability, the derivate has to be defined by convergence of nets rather than convergence of sequences. Also the possibility of noncompact domains of integration requires a different definition of the majorant.

Throughout $P$ is a topological space and $P^{\sim}=P \cup(\infty)$ is a onepoint compactification of $P$. If $A \subset P^{-}, A^{-}$and $A^{*}$ denote the closure of $A$ in $P$ and $P^{\sim}$, respectively. For $x \in P^{\sim}, \Gamma_{x}$ is a local base at $x$ in $P^{\sim}$ (see [6, p. 50]).

Let $\sigma$ be a nonempty system of subsets of $P$ such that for every $A, B \in \sigma, A \cap B \in \sigma$ and $A-B=\cup_{i=1}^{n} C_{i}$ where $C_{1}, \cdots, C_{n}$ are disjoint sets from $\sigma .^{2}$ We shall assume that $\Gamma_{x} \subset \sigma$ for every $x \in P$ and that for each $U \in \Gamma_{\infty}$ there are disjoint sets $U_{1, \infty}, \ldots, U_{p, \infty}$ from $\sigma$ such that $U \cap P=\cup_{i=1}^{p} U_{i, \infty}$ where the integer $p \geqq 1$ is independent of $U$.

${ }_{1}^{1}$ This research was partially supported by a University of California summer faculty fellowship.

${ }^{2}$ Such a system $\sigma$ is called a prering in [2]. The sets from $\sigma$ generate the ring over which the integral will be defined. Since our main task is to define a nonabsolutely convergent integral, it should be mentioned here that the system $\sigma$ must not be too large; e.g., if $\sigma$ is a $\sigma$-ring, then usually only an absolutely convergent integral is obtained. We also note that more general systems than prerings can be used for $\sigma$, e.g., a system of all simplexes in a given Euclidean space. 
The algebra generated by $\sigma$ is denoted by $\sigma^{\wedge}$; clearly $P \in \sigma^{\wedge}$. If $\delta$ is a collection of subsets of $P$ and $A \subset P^{-}$, we let $\delta_{A}=\{B \in \delta: B \subset A\}$. A system $\delta \subset \sigma$ is said to be semihereditary if and only if $\sigma_{0} \cap \delta \neq \varnothing$ for every finite disjoint collection $\sigma_{0} \subset \sigma$ whose union belongs to $\delta$. A system $\delta \subset \sigma$ is said to be stable if and only if $\varnothing \notin \delta$ and for every $A \in \delta$ and every $x \in P^{-}$there is a $U \in \Gamma_{x}$ such that $\delta_{A-U} \neq \varnothing$.

By a function we shall mean an extended real-valued function. A function $F$ defined on $\delta C \sigma^{\wedge}$ is said to be superadditive or additive whenever $F\left(\cup_{i-1}^{n} A_{i}\right) \geqq \sum_{i=1}^{n} F\left(A_{i}\right)$ or $F\left(\cup_{i=1}^{n} A_{i}\right)=\sum_{i=1}^{n} F\left(A_{i}\right)$, respectively, for every disjoint collection $A_{1}, \cdots, A_{n}$ from $\delta$ for which $\cup_{i=1}^{n} A_{i} \in \delta$ and $\sum_{i=1}^{n} F\left(A_{i}\right)$ has meaning.

Semihereditary and stable systems and their connection with superadditive functions were investigated in [11], [12], and [15].

From now on we shall assume that there is given a nonnegative additive function $G$ defined on $\sigma^{\wedge}$ and such that $G(U)<+\infty$ for every $U \in U\left\{\Gamma_{x}: x \in P\right\}$. Intuitively, $G$ is a finitely additive locally finite measure in $P$.

With every point $x \in P^{-}$we associate a certain family $\kappa_{x}$ of nets $\left\{B_{U}, U \in \Gamma, C\right\} \subset \sigma$ where $\Gamma$ is a cofinal subset of $\Gamma_{x}$ (see [6, Chapter 2]). The collection $\kappa=\left\{\kappa_{x}: x \in P^{-}\right\}$is called a convergence (or sometimes a derivation basis-see $[4,1.1])$. For $x \in P^{-}$and $\delta \subset \sigma, \kappa_{x}(\delta)$ $=\left\{\left\{B_{U}\right\} \in \kappa_{x}:\left\{B_{U}\right\} \subset \delta\right\}$.

Throughout we shall assume that the convergence $\kappa$ satisfies the following conditions: ${ }^{3}$

$\varkappa_{1}$. For every $x \in P,\left\{U, U \in \Gamma_{x}, \subset\right\} \in \kappa_{x}$ and for every integer $i, 1 \leqq i \leqq p,\left\{U_{i, \infty}, U \in \Gamma_{\infty}, C\right\} \in \kappa_{\infty}$.

$\mathfrak{K}_{2}$. If $x \in P^{-}$and $\left\{B_{U}, U \in \Gamma, C\right\} \in \kappa_{x}$, then for every $V \in \Gamma_{x}$ there is a $U_{V} \in \Gamma$ such that $B_{U} \subset V$ for all $U \in \Gamma$ for which $U \subset U_{V}$.

$\varkappa_{3}$. If $x \in P^{-},\left\{B_{U}\right\}_{U \in \Gamma} \in \kappa_{x}$, and $\Gamma^{\prime}$ is a cofinal subset of $\Gamma$, then also $\left\{B_{U}\right\}_{v \in \Gamma^{\prime}} \in \kappa_{x}$.

$\mathscr{K}_{4}$. If $x \in P^{\sim},\left\{B_{U}\right\} \in \kappa_{x}$, and $A \in \sigma$, then also $\left\{B_{U} \cap A\right\} \in \kappa_{x}$.

$\mathcal{K}_{5}$. If $\delta \subset \sigma$ is a nonempty semihereditary, stable system, then the set $\left\{x \in P^{-}: \kappa_{x}(\delta) \neq \varnothing\right\}$ is uncountable.

If for every $x \in P^{*}, \kappa_{x}$ consists of all nets $\left\{B_{U}\right\}$ which satisfy condition $\varkappa_{2}$, then the convergence $\kappa$ is called the natural convergence and is denoted by $\kappa^{0}$. It follows from $[12,2.4]$ that the natural convergence already satisfies all conditions $\mathfrak{K}_{1}-\mathfrak{K}_{5}$. Therefore, conditions

3 The abstract notion of a convergence is the basis for the whole theory. Proposition 4 indicates that we should use a convergence $\kappa$ with $\kappa_{x}$ as small as possible. On the other hand, it is plain that if the $\kappa_{x}$ are too small, we shall not obtain any integral at all. Conditions $\mathcal{K}_{1}-\mathcal{K}_{b}$ form a set of minimal requirements which a convergence $\kappa$ must satisfy in order to give a meaningful integral. 
$\varkappa_{1}-\mathfrak{K}_{5}$ are not contradictory and it was shown in [17], that they are also independent. Other useful examples of convergences which satisfy conditions $\mathfrak{K}_{1}-\mathfrak{K}_{5}$ can be found in [9], [10], and [17].

Let $x \in P^{-}, A \subset P$, and let $F$ be a function on $\sigma_{A}$. We call the number $f F(x, A)=\inf \left\{\lim \inf F\left(B_{U}\right):\left\{B_{U}\right\} \in \kappa_{x}\left(\sigma_{A}\right)\right\}$ the lower limit of $F$ at $x$ relative to $A$ and the number $* F(x, A)=*(F / G)(x, A)^{4}$ the lower derivate of $F$ at $x$ relative to $A$.

Let $A \in \sigma$ and let $f$ be a function on $A^{-}$. A superadditive function $M$ on $\sigma_{A}$ is said to be a majorant of $f$ on $A$ if and only if there is a countable set $Z_{M} \subset A^{-}$such that $\sharp(-G)(x, A) \geqq 0$ for all $x \in Z_{M}$, $t M(x, A) \geqq 0$ for all $x \in Z_{M} \cup(\infty)$, and $-\infty \neq * M(x, A) \geqq f(x)$ for all $x \in A^{-}-Z_{M}$. The number $I_{u}(f, A)=\inf M(A)$ where the infimum is taken over all majorants of $f$ on $A$ is called the upper integral of $f$ over $A$.

Proposition 1. Let $A \in \sigma$ and let $f$ be a function on $A^{-}$. If $f \geqq 0$ then $I_{u}(f, A) \geqq 0$.

Proposition 2. Let $A \in \sigma$ and let $f$ be a function on $A^{-}$. Then the function $I_{u}(f, \cdot)$ is additive on $\sigma_{A}$.

Proposition 3. Let $A \in \sigma$ and let $\left\{f_{n}\right\}$ be an increasing sequence of functions defined on $A^{-}$. If $I_{u}\left(f_{1}, A\right)>-\infty$ then

$$
\lim I_{u}\left(f_{n}, A\right)=I_{u}\left(\lim f_{n}, A\right) .
$$

Definition. Let $A \in \sigma^{\wedge}$ and let $f$ be a function on $A^{-}$. Choose disjoint sets $A_{1}, \cdots, A_{n}$ from $\sigma$ such that $\bigcup_{i=1}^{n} A_{i}=A$ and set

$$
I_{u}(f, A)=\sum_{i=1}^{n} I_{u}\left(f, A_{i}\right) \text {. }
$$

When $I_{u}(f, A)=-I_{u}(-f, A) \neq \pm \infty$ this common value is called the integral of $f$ over $A$ and it is denoted by $I(f, A)$.

By $[12,(1.1)]$ the sets $A_{1}, \cdots, A_{n}$ always exist and by Proposition 2 the value of $I_{u}(f, A)$ is independent of their choice. Thus the previous definition extends the original meaning of the upper integral. If $A \in \sigma^{\wedge}, \mathfrak{B}(A)$ denotes the family of all functions $f$ defined on $A^{-}$for which the integral $I(f, A)$ exists and $\mathfrak{B}_{0}(A)=$ $\{f \in \mathfrak{P}(A):|f| \in \mathfrak{B}(A)\}$.

ThEOREM 1. If $A \in \sigma^{\wedge}$ and $f \in \mathfrak{B}(A)$, then $f \in \mathfrak{P}(B)$ for every $B \in \hat{\sigma_{A}}$.

ThEOREM 2. Let $A \in \sigma^{\wedge}, f, g \in \mathfrak{P}(A)$ and let $a, b$ be real numbers. If

- We let $a / 0=+\infty$ for $a \geqq 0, a / 0=-\infty$ for $\mathrm{a}<0$, and $a /( \pm \infty)=0$. 
$h(x)=a f(x)+b g(x)$ for all $x \in A^{-}$for which $a f(x)+b g(x)$ has meaning, then $h \in \mathfrak{P}(A)$ and

$$
I(h, A)=a I(f, A)+b I(g, A) .
$$

Moreover, if $f, g \in \mathfrak{B}_{0}(A)$ then also $h \in \mathfrak{P}_{0}(A)$.

Corollary. Let $A \in \sigma$ and $f, g \in \mathfrak{P}_{0}(A)$. Then $\max (f, g) \in \mathfrak{B}_{0}(A)$ and $\min (f, g) \in \mathfrak{B}_{0}(A)$. Moreover, if $h \in \mathfrak{B}(A)$ and $f \leqq h \leqq g$, then $h \in \mathfrak{P}_{0}(A)$.

Theorem 3. Let $A \in \sigma, f, g, h_{n} \in \mathfrak{P}(A)$, and let $f \leqq h_{n} \leqq g$ for $n$ $=1,2, \cdots$. If $\lim h_{n}=h$ then $h \in \mathfrak{B}(A)$ and

$$
I(h, A)=\lim I\left(h_{n}, A\right) .
$$

Example 1. Let $P$ be $r$-dimensional Euclidean space and let $\sigma$ be the system of all half-open intervals (bounded, unbounded, or degenerate). For $x \in P^{\sim}$ let $\kappa_{x}$ consist of all sequences $\left\{K_{n}\right\}_{n=1}^{\infty} \subset \sigma$ which satisfy condition $\mathcal{K}_{2}$ and such that either $x \in \bigcap_{n=1}^{\infty} K_{n}^{*}$ or $K_{n}=\varnothing$ for all sufficiently large $n$. Then the convergence $\kappa$ satisfies conditions $\mathscr{K}_{1}-\mathscr{K}_{5}$ (see $[12,3.2]$ ) and the integral $I$ coincides with the integral defined by Mařik in [7]. In particular, if $r=1$ and if $G$ is the restriction of the Lebesgue measure, then the integral $I$ coincides with the classical Perron integral (see [8] or [18]).

ExAmple 2. Let $P$ be the discrete space of positive integers, let $\sigma$ consist of the empty set, singletons $(n)$, and intervals $[n,+\infty), n$ $=1,2, \cdots$, and let $\kappa=\kappa^{0}$ be the natural convergence. If $G$ is the counting measure on $\sigma$, then $f \in \mathfrak{B}(P)$ if and only if the series $\sum_{n=1}^{\infty} f(n)$ is conditionally convergent; $I(f, P)=\sum_{n=1}^{\infty} f(n)$ when either side has meaning.

Given another convergence $\kappa^{\prime}=\left\{\kappa_{x}^{\prime}: x \in P^{-}\right\}$which satisfies conditions $\mathfrak{K}_{1}-\mathfrak{K}_{5}$, we can introduce the symbols $I^{\prime}$ and $\mathfrak{P}^{\prime}$ the meaning of which is obvious. The connection between $I, \mathfrak{P}$ and $I^{\prime}, \mathfrak{P}^{\prime}$ is given by the following proposition.

Proposition 4. Let $\kappa_{x}^{\prime} \subset \kappa_{x}$ for all $x \in P^{-}$. Then for every $A \in \sigma, \mathfrak{P}(A)$ $\subset \mathfrak{P}^{\prime}(A)$ and $I(f, A)=I^{\prime}(f, A)$ for all $f \in \mathfrak{P}(A)$.

A point $x \in P^{\sim}$ is said to be simple if and only if $\Gamma_{x}=\left\{U_{n}\right\}_{n=1}^{\infty}, \sigma_{U_{1}}$ $=\hat{\sigma}_{U_{1}}$, and for every $\left\{B_{n}\right\}$ and $\left\{C_{n}\right\}$ from $\kappa_{x}\left(\sigma_{U_{1}}\right)$ also $\left\{B_{n} \cup C_{n}\right\}$ and $\left\{B_{n}-C_{n}\right\}$ belong to $\kappa_{x}\left(\sigma_{U_{1}}\right)$. Notice that if $\sigma=\sigma^{\wedge}$ and $\kappa=\kappa^{0}$ is the natural convergence, then $x \in P^{-}$is simple whenever $\Gamma_{x}=\left\{U_{n}\right\}_{n=1}^{\infty}$.

TheOREM 4. Let $A \in \sigma^{\wedge}$ and let $f$ be a function on $A^{-}$. Let $x \in P^{\sim}$ be a simple point at which $P^{-}$is Hausdorff and let either $x \notin A^{-}$or

5 This means that for every $y \in P^{\sim}-(x)$ there are disjoint sets $U \in \Gamma_{x}$ and $V \in \Gamma_{y}$. 
$+(-G)(x, A) \geqq 0$. Further suppose that $f \in \mathfrak{B}(B)$ for every $B \in \hat{\sigma_{A}}$ for which $x \notin B^{\sim}$ and that

$$
\lim I\left(f, A-B_{n}\right)=c \neq \pm \infty
$$

for every $\left\{B_{n}\right\} \in \kappa_{x}$ for which $x \notin\left(A-B_{n}\right)^{\sim}, n=1,2, \cdots$. Then $f \in \mathfrak{B}(A)$ and $I(f, A)=c$.

For the remainder of this paper we shall assume that $P$ is a locally compact Hausdorff space. Unless otherwise specified our terminology concerning measures is that of [3]. If $A \subset P, \chi_{A}$ denotes the characteristic function of $A$. Let $\mathfrak{I}$ be the family of all sets $A \subset P$ for which $\chi_{A} \cap_{C} \in \mathfrak{B}_{0}(P)$ for every compact set $C \subset P$. For $A \in \mathfrak{I}$ we let $\iota(A)$ $=I_{u}\left(\chi_{A}, P\right)$.

TheOREM 5. The triple $(P, \mathfrak{T}, \iota)$ is a complete measure space and $\mathfrak{I}$ contains all open subsets of $P$. The measure $\iota$ is inner regular on open sets and outer regular and finite on compact sets. Moreover, if $C \subset P$ is compact, then $\iota(C)=\inf G(A)$ where the infimum is taken over all sets $A \in \sigma^{\wedge}$ whose interior contains $C .^{\circ}$

TheOREM 6. A function $f$ belongs to $\mathfrak{B}_{0}(P)$ if and only if the Lebesgue integral $\int_{P} f d \iota$ exists; $I(f, P)=\int_{P} f d \iota$ when either side has meaning. Moreover, if for every $A \in \sigma$ and for every function $f$ on $A$ - the upper integral $I_{u}(f, A)$ is independent of values which $f$ takes on $A^{-}-A$, then $\sigma \subset \mathfrak{T}$ and a function $f$ belongs to $\mathfrak{P}_{0}(A), A \in \sigma$, if and only if the Lebesgue integral $\int_{A} f d \iota$ exists; $I(f, A)=\int_{A} f d \iota$ when either side has meaning.

For $A \subset P$ let $\iota_{0}(A)=\inf \iota(U)$, where the infimum is taken over all open sets $U \subset P$ for which $A \subset U$. It is easy to see that $\iota_{0}$ is an outer measure in $P$ and we shall denote by $\mathfrak{T}_{0}$ the family of all $\iota_{0}$-measurable subsets of $P$.

THEOREM 7. The triple $\left(P, \mathfrak{T}_{0}, \iota_{0}\right)$ is a complete measure space and the measure $\iota_{0}$ is regular. ${ }^{7}$ Moreover, if $(P, \mathfrak{A}, \mu)$ is a measure space with a regular measure $\mu, \sigma \subset \mathfrak{A}$, and $G(A)=\mu(A)$ for every $A \in \sigma$ for which $A^{-}$ is compact, then $\mathfrak{A} \subset \mathfrak{I}_{0}$ and $\mu(A)=\iota_{0}(A)$ for every $A \in \mathfrak{A}$.

Theorem 8. Always $\mathfrak{T}_{0} \subset \mathfrak{T}$ and $\iota_{0}(A)=\iota(A)$ for every set $A \in \mathfrak{I}_{0}$ which is $\iota_{0}-\sigma$-finite. If $P$ is paracompact then $\iota_{0}(A)=\iota(A)$ for every $A \in \mathfrak{I}_{0}$.

ThEOREM 9. Suppose that $\sigma \subset \mathfrak{T}_{0}$ and that $G(A)=\iota_{0}(A)$ for every $A \in \sigma$

- The third part of Theorem 5 is due to W. J. Wilbur.

7 See [5, (12.39), p. 177]. 
for which $A^{-}$is compact. Let $A \in \sigma$ and let $f$ be a function on $A^{-}$for which the Lebesgue integral $\int_{A} f d \iota_{0}$ exists. If $A^{-}-A$ is $\iota_{0}-\sigma$-finite then $f \in \mathfrak{B}_{0}(A)$ and $I(f, A)=\int_{A} f d \iota_{0}$.

CoRollary. Let $\sigma \subset \mathfrak{I}_{0}$ and let $G(A)=\iota_{0}(A)$ for every $A \in \sigma$ for which $A^{-}$is compact. If $\iota_{0}$ is $\sigma$-finite then for every $A \in \sigma$ and for every function $f$ on $A^{-}$the upper integral $I_{u}(f, A)$ is independent of values which $f$ takes on $A^{-}-A$.

Let $\kappa=\kappa^{0}$ be the natural convergence. It was proved in [17] that in this case $(P, \mathfrak{I}, \iota)=\left(P, \mathfrak{I}_{0}, \iota_{0}\right)$ and every function $f \in \mathfrak{B}(A)$ is ı-measurable. Moreover, if $\sigma=\sigma^{\wedge}$ then $\mathfrak{B}(A)=\mathfrak{B}_{0}(A)$ for every $A \in \sigma$.

ExAmple 3. Let $P=(-\infty,+\infty)$ and let $\sigma$ consist of all intervals which either do not contain 0 in their closures or are of the form $(-\epsilon, \epsilon)$ where $\epsilon>0$. Let $\kappa_{x}=\kappa_{x}^{0}$ for $x \neq 0$ and let $\kappa_{0}$ consist of the net $\{(-\epsilon, \epsilon)\}_{\epsilon>0}$ and the trivial net $\{\varnothing\}$. Finally, let $G$ be the restriction of the Lebesgue measure. Then $\kappa$ satisfies conditions $\mathfrak{K}_{1}-\mathfrak{K}_{5}$ and $f$ belongs to $\mathfrak{B}_{0}(P)$ if and only if the Lebesgue integral $\int_{-\infty}^{+\infty} f$ exists; $I(f, P)=\int_{-\infty}^{+\infty} f$ when either side has meaning. On the other hand, $f$ belongs to $\mathfrak{B}(P)$ if and only if the Cauchy principle value

$$
\text { (pv) } \int_{-\infty}^{+\infty} f=\lim _{\epsilon \rightarrow 0+}\left(\int_{-\infty}^{-\epsilon} f+\int_{\epsilon}^{+\infty} f\right)
$$

exists; $I(f, P)=(p v) \int_{-\infty}^{+\infty} f$ when either side has meaning. Notice that 0 is not a simple point of $P$.

Complete proofs of the results stated here will be given in [13], [14] and [16].

\section{REFERENCES}

1. H. Bauer, Der Perronsche Intergralbegriff und seine Beziehung zum Lebesgueschen, Monatsh. Math. Phys. 26 (1915), 153-198.

2. W. M. Bogdanowicz, A generalization of the Lebesgue-Bochner-Stieltjes integral and a new approach to the theory of integration, Proc. Nat. Acad. Sci. U.S.A. 53 (1965), 492-498.

3. P. R. Halmos, Measure theory, Van Nostrand, New York, 1950.

4. C. A. Hayes and C. Y. Pauc, Full individual and class differentiation theorems in their relations to halo and Vitali properties, Canad. J. Math. 7 (1955), 221-274.

5. E. Hewitt and K. Stromberg, Real and abstract analysis, Springer-Verlag, New York, 1965.

6. J. L. Kelley, General topology, Van Nostrand, New York, 1955.

7. J. Mařik, Foundation of the theory of an integral in Euclidean spaces. I-III, Casopis Płst. Mat. 77 (1951), 1-51, 125-145, 267-300.

8. O. Perron Über den Integralbegriff S.-B. Heidelberger Acad. Wiss. Math.Natur. Kl. 16 (1914).

9. W. F. Pfeffer, The Perron integral in topological spaces, Casopis Pest. Mat. 88 (1963), 322-348. 
10. - On a definition of the integral in topological spaces. I, II, Casopis Pest. Mat. 89 (1964), 129-147, 257-277.

11. - A note on the lower derivate of a set function and semihereditary systems of sets, Proc. Amer. Math. Soc. 18 (1967), 1020-1025.

12. - On the lower derivate of a set function, Canad. J. Math. 20 (1968), 1489-1498.

13. - The integral in topological spaces. I and II (to appear).

14. - Singular integrals are Perron integrals of a certain type (to appear).

15. W. F. Pfeffer and W. J. Wilbur, $A$ note on cluster points of a semihereditary stable system of sets, Proc. Amer. Math. Soc. 21 (1969).

16. - On the measurability of Perron integrable functions (to appear).

17. W. J. Wilbur, On non-absolute integration in topological spaces, Ph.D. thesis, University of California, Davis, Calif.

18. S. Saks, Theory of the integral, Hafner, New York, 1937.

University of California, Davis, California 95616

\section{ON GENERALIZED COMPLETE METRIC SPACES}

BY JAMES D. STEIN, JR.

Communicated by J. B. Diaz, October 3, 1968

The following remarks are of interest in connection with the research announcement [1]:

Lemma. A generalized metric space is the disjoint union of metric spaces such that each metric space is infinitely distant from every other metric space.

Proof. Note that $d(x, y)<\infty$ is an equivalence relation, and the equivalence classes obtained are metric spaces. Also, if the generalized space is complete, so is each metric space. Q.E.D.

Let $M=\mathrm{V}_{\alpha e A} M_{\alpha}$ denote the above partitioning. The Banach contraction principle becomes

Proposition 1. Let $T$ be a strict contraction of a generalized complete metric space $M=V_{\alpha \in A} M_{\alpha}, 0 \leqq q<1, d(x, y)<\infty \Rightarrow d(T x, T y) \leqq q d(x, y)$. For each $\alpha \in A, \exists \beta \in A$ such that $T\left(M_{\alpha}\right) \subseteq M_{\beta}$. There is a unique periodic point of order $n$ in each $M_{\alpha}$ such that $T^{n}\left(M_{\alpha}\right) \subseteq M_{\alpha}$.

Proof. Let $x, y \in M_{\alpha}, T x \in M_{\beta}$. Then $d(x, y)<\infty \Rightarrow d(T x, T y)<\infty$ $\Rightarrow T y \in M_{\beta}$. Since $T^{n}$ is a strict contraction of the complete metric space $M_{\alpha}$, it has a unique fixed point, which is a periodic point of order $n$ for T. Q.E.D.

The local contraction principle becomes 